\title{
Ceratoprótese de Boston
}

\author{
Boston Keratoprosthesis
}

Sérgio Kwitko ${ }^{1}$, Andressa Prestes Stolz ${ }^{2}$

\begin{abstract}
RESUMO
Uma breve revisão de resultados e complicações da ceratoprótese (KPro) de Dohlman-Doane tipo 1. A KProDohlman-Doane é atualmente uma boa opção em casos graves de cegueira corneana, para os quais o transplante penetrante de córnea não apresenta bom prognóstico. As evoluções no design da KPro e as melhorias no procedimento cirúrgico, aliados a obtenção de condições favoráveis da superfície ocular pré-operatórias, garantem bons resultados da KPro em doenças não autoimunes. As principais complicações da KPro são membrana inflamatória retroprotética de fibrina, glaucoma, necrose corneana, vitreíte e infecção (ceratite e endoftalmite). Acompanhamento constante com exames pós-operatórios periódicos é necessário para monitorar e prevenir complicações sérias.
\end{abstract}

Descritores:Doenças da córnea/cirurgia; Próteses e implantes; Implantação de próteses

\begin{abstract}
A short review of type 1 Dohlman-DoaneKeratoprosthesis (KPro) addresses results and complications. Dohlman-DoaneKPro is today a good option for cases of corneal blindness with poor prognosis for traditional penetrating keratoplasty. KPro design and surgical improvements along with proper preoperative ocular surface condition allows good results in non-immune diseases. Main complications of this KPro areinflammatory retroprosthetic membrane, glaucoma, corneal melting, vitreitis, and infection (keratitis and endophthalmitis). Comprehensive long-term postoperative follow-up examinationsare needed to monitor for and prevent serious complications.

Keywords: Corneal diseases/surgery; Prosthesesandimplants; Prosthesisimplantation
\end{abstract}

\footnotetext{
${ }^{1}$ Doutor, professor do Programa de pós-graduação em Cirurgia da Universidade Federal do Rio Grande do Sul (UFRGS) - Porto Alegre (RS), Brasil;

${ }^{2}$ Chefe do Serviço de Oftalmologia do Hospital da Brigada Militar de Porto Alegre - Porto Alegre (RS), Brasil.

Os autores declaram não haver conflitos de interesse

Recebido para publicação em: 18/8/2011 - Aceito para publicação em: 26/9/2011
}

Rev Bras Oftalmol. 2012; 71 (6): 403-6 


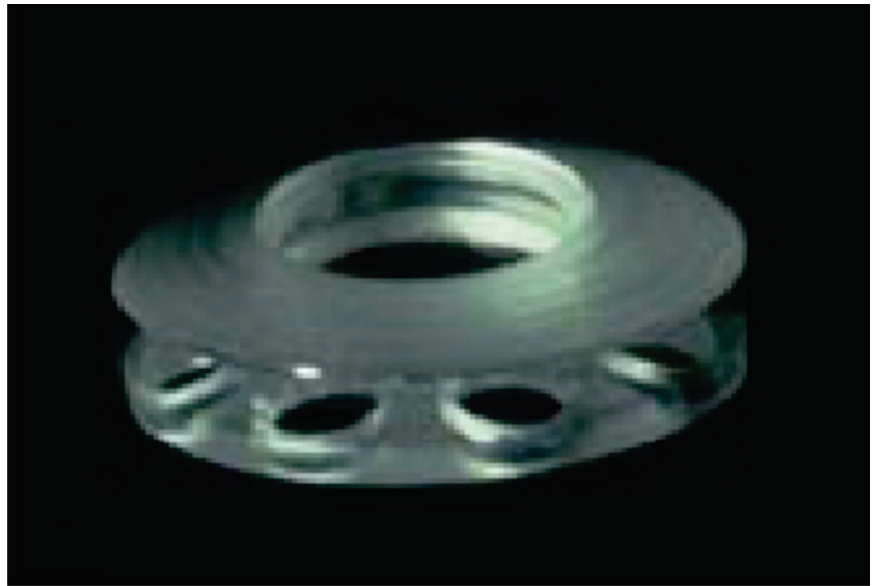

Figura 1: Aspecto da Boston K-Pro tipo 1

Há muito que se almeja confeccionar uma córnea artificial, principalmente com os objetivos de não necessitar um doador humano, anular a possibilidade de rejeição do transplante e não necessitar imunossupressão sistêmica nos casos de alto risco de rejeição. Contudo, ainda não se conseguiu desenvolver uma córnea artificial ideal que atinja esses objetivos e que ao mesmo tempo seja capaz de uma integração perfeita com o tecido receptor, sem riscos de extrusão ou complicações.

O que já se consegue, entretanto, é a substituição da córnea por lentes artificiais - as chamadas ceratopróteses - que podem ser confeccionadas de material biocompatível, numa tentativa de integração com o tecido vivo e consequentemente menor índice de extrusão, ou de material sintético, como o Polimetilmetacrilato (PMMA) e o hidrogel que, em casos específicos, detém atualmente um índice de sucesso satisfatório a médio prazo.

Os modelos de ceratopróteses mais utilizados atualmente são: 1.Cératoprótese de Strampelli-Falcinelli ou ósteo-odontoceratoprótese, na qual é necessário o implante da ceratoprótese de acrílico em um dente do paciente para posterior implante na córnea receptora, cirurgia trabalhosa realizada em duas etapas, com aproximadamente 3 meses de intervalo ${ }^{(1,2)}$;

2. CeratpróteseAlphaCor, produzida na Austrália, semelhante a uma lente de contato hidrofílica, mas ainda com muitas limitações, principalmente nos casos deolho seco, leucoma pós-herpético e doenças imunes de membranas $\operatorname{mucosas}^{(3)}$;

3. Ceratoprótese de Dohlman-Doane, produzida em Boston, EUA, a qual foi uma das que mais evoluiu nos últimos anos, apresentando atualmente um índice de sucesso de $90 \%$ em casos de doenças não imunológicas, e de $50 \%$ em casos de doenças imunes de membranas $\operatorname{mucosas}^{(4)}$.

Em casos de múltiplos transplantes de córnea, a cada novo enxerto corneano aumenta o risco de rejeição e de complicações como glaucoma e edema macular cistóide, além do tempo médio de sobrevivência do enxerto diminuir ${ }^{(5)}$. Além disto, em casos de doenças severas da superfície ocular, como queimadura alcalina grave bilateral e doenças imunes de membranas mucosas, o prognóstico do transplante de córnea é muito reservado, mesmo com imunossupressão sistêmica ${ }^{(6,7)}$.

A ceratoprótese de Dohlman-Doane ou Boston Kerato-

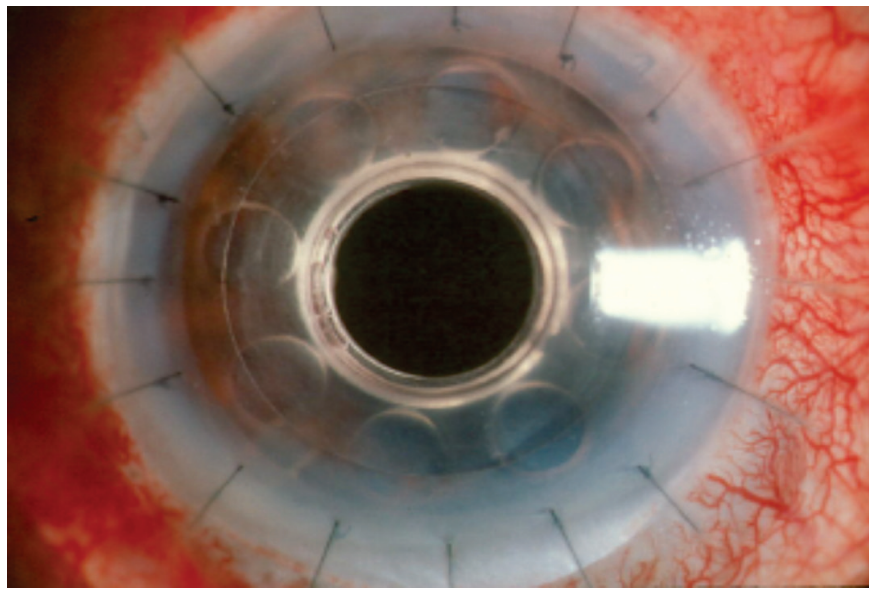

Figura 2: Aspecto pós-operatório

prosthesis (K-Pro) tem sido uma importante alternativa nos casos de cegueira corneana para os quais o transplante penetrante de córnea não apresenta bom prognóstico ${ }^{(4,6)}$.

Uma importantevantagem da ceratoprótese de DohlmanDoaneem pacientes com transplantes de alto risco é que não há necessidade, na grande maioria dos casos, de imunossupressão sistêmica. Dohlman tem recentemente proposto imunossupressão sistêmica para pacientes com doenças de membranas mucosas ${ }^{(8)}$.

Vale lembrar que os casos de queimadura alcalina e de alto risco de rejeição, como múltiplos transplantes prévios, apresentam melhor prognóstico que os pacientes com doenças de membranas mucosas, como Stevens-Johnson, Lyell e penfigóide ocular cicatricial ${ }^{(9)}$.

Dois tipos de K-Pro de Dohlman-Doane foram desenvolvidos, uma para pacientes com relativa boa hidratação da superfície ocular e fechamento palpebral (tipo 1) e outra para casos de severo olho seco, usada trans-palpebralmente (tipo 2). Ambas podem ser produzidas para olhos afácicos, com poder dióptrico calculado pelo diâmetro axial, ou para olhos pseudofácicos, com poder único ${ }^{(10,11)}$.

Esta ceratoprótese é produzida em PMMA com uma zona óptica central de $3.35 \mathrm{~mm}$ de diâmetro, e uma plataforma posterior de $0.9 \mathrm{~mm}$ de espessura e $7.0 \mathrm{~mm}$ ou $8.5 \mathrm{~mm}$ de diâmetro, com 8 a 16 fenestrações para permitir uma melhor nutrição da córnea doadora pelo humor aquoso (Figura 1). Ela é montada em uma córnea doadora humana (Figura 2), sendo uma cirurgia acessível ao cirurgião de córnea, com uma curva de aprendizado pequena.

A cirurgia é de mais fácil aprendizado que as outras ceratopróteses e é confeccionada em um só tempo cirúrgico, ao contrário da ósteo-odontoceratoprótese.

O procedimento é geralmente realizado com anestesia peribulbar, em caráter ambulatorial, como um transplante penetrante regular, onde se faz uma diferença receptor-doador de pelo menos $1 \mathrm{~mm}$, e se deixa o paciente pseudofácico ou afácico, mesmo que o cristalino seja transparente, devido à grande incidência pósoperatória de catarata e consequente dificuldade da extração da catarata através de uma transparência corneana central de somente $3.35 \mathrm{~mm}^{(4)}$.

Ao final da cirurgia, é importante que se deixe dexametasona na câmara anterior e uma lente terapêutica de diâmetro grande (de $16.0 \mathrm{~mm}$ ), para reduzir o risco de necrose corneana e extrusão da ceratoprótese ${ }^{(5)}$.

No pós-operatório, corticóide tópico e sistêmico são usados por um período médio de 30 dias. O paciente necessita usar continuadamente antibioticoprofilaxia tópica, preferentemente 
uma quinolona de $4^{\mathrm{a}}$ geração ou vancomicina ${ }^{(12)}$. Nossa preferência é pela gatifloxacina, por apresentar menor toxicidade epitelial que a moxifloxacina ${ }^{(13)}$ e que a vancomicina.

Além disso, o paciente deve manter o uso contínuo de lubrificantes sem conservantes, medroxiprogesterona e lente de contato terapêutica, que reduzem o risco de necrose corneana e conseqüente extrusão da ceratoprótese ${ }^{(14,15)}$.

Este procedimento vem ganhando significativa aceitação e popularidade desde que foi introduzida na década de 60 por ClaesDohlman, devido a uma série de fatores,quais sejam:

1) Melhora da técnica cirúrgica do transplante de córnea;

2) Melhora da qualidade do tecido doador;

3) Melhora da preservação endotelial da córnea doadora;

4) Utilização de prato posterior de titânio;

5) Maior número de fenestrações do prato posterior para melhor nutrição da córnea doadora;

6) Melhores viscoelásticos;

7) Melhores antibióticos profiláticos;

8) Melhor profilaxia da necrose (lentes de contato terapêuticas grandes, melhor tratamento do olho seco, medroxiprogesterona);

9) Melhor entendimento e preparo da superfície ocular prévio ao implante da ceratoprótese;

10)Melhor controle clínico ou cirúrgico do glaucoma.

É muito importante lembrar que o adequado tratamento e preparo prévio da superfície ocular é fundamental para a melhora do prognóstico da ceratoprótese.

Isto inclui:

a) um intenso tratamento do olho seco, comum em pacien tes com doenças da superfície ocular, com oclusão per manente dos pontos lacrimais, lágrimas artificiais sem conservantes, soro autólogo, Ômega-3 e transplante de glândulas salivares de acordo com a gravidade do caso $^{(14,16)}$;

b) correção das alterações palpebrais, também muito comum nestes casos, tais como entrópio, triquíase, distiquíase, encurtamento do fundo-de-saco etc ${ }^{(16,17)}$;

c) melhora anatômica e funcional do limbo, através de transplante de limbo ${ }^{(16,18-20)}$;

d) redução do processo inflamatório da superfície ocular através do transplante de membrana amniótica ${ }^{(16,21)}$.

A ceratoprótese de Dohlman-Doane pode ser implantada apenas em olhos pseudofácicos ou afácicos, portanto a facectomia é necessária independentemente do grau de transparência do cristalino. Como é comum nestes pacientes a presença de glaucoma, é frequente a associação no mesmo tempo cirúrgico do implante de válvula de Ahmed. Este último pode também ser realizado prévia ou posteriormente à ceratoprótese.

A literatura relata uma significativa parcela de pacientes com importante melhora da acuidade visual (AV) e da qualidade de vida destes pacientes que até então eram cegos e pouco se podia fazer por eles. Em 1 ano de pós-operatório 57\% a $83 \%$ obtém AV de 20/200 ou melhor ${ }^{(22-25)}$.

Apesar dos resultados mostrarem-se favoráveis a curto prazo, existem diversas complicações que ameaçam o prognóstico a longo prazo desses implantes. Isto inclui extrusão da prótese, formação de membrana retroprotética, necrose corneana, crescimento epitelial intracameral, glaucoma, descolamento de retina e endoftalmite ${ }^{(12)}$.

Isto tem gerado importantes problemas psicológicos nes- tes pacientes, levando inclusive ao suicídio, já que tiveram reabilitação visual temporária e em muito casos sem possibilidade de uma nova recuperação. Faz-se, necessário, portanto,um acompanhamento psicológico/psiquiátrico destes pacientes.

A taxa de retenção da ceratoprótese a curto prazo, até 12 meses de seguimento, é muito alta, relatada por $83 \%$ a $100 \%$ ${ }^{25)}$. Entretanto, após 2 anos, cai para $60 \%$ nas poucas séries relatadas com acompanhamento a longo prazo ${ }^{(22)}$.

Necrose da interface ceratoprótese-córnea também é um dos maiores problemas a médio e longo prazos. O uso permanente de soro autólogo, lente de contato terapêutica e medroxiprogesterona tem reduzido significativamente a incidência desta complicação. Esta complicação ocorre de 1\% a $29 \%$ dos $\operatorname{casos}^{(4,6,26)}$. O tratamento com enxerto de membrana amniótica e recobrimento conjuntival é bem-sucedido na maioria dos $\operatorname{casos}^{(5)}$, entretanto há necessidade da troca do botão doador em uma parcela significativa de casos.

Ceratite e endoftalmite micótica é um risco potencial nestes casos, devido à presença permanente de material não biocompatível (PMMA) na superfície ocular, o uso permanente de lente de contato terapêutica e antibiótico tópico profilático. Ceratite micótica é relatada de 3\% a 17\% dos casos, motivo pelo qual Dohlman propôs recentemente um ciclo trimestral de Anfotericina-B tópica ${ }^{(27)}$, associado ao já bem estabelecido uso de vancomicina colírio profilático ${ }^{(28,29)}$.

Formação de membrana retroprotética é uma complicação também comum, relatada em 25 a $65 \%$ dos $\operatorname{casos}^{(4,7,22-25)}$. O tratamento com YAG laser é geralmente bem-sucedido ${ }^{(11)}$.

Opacidade de cápsula posterior, relatada em 2\% a $15 \%$ dos $\operatorname{casos}^{(23-25)}$, tem prognóstico bem favorável com a realização da capsulotomia posterior com YAG laser.

Vitreíte estéril é uma complicação também significativa, ocorrendo de $3 \%$ a $10 \%$ dos $\operatorname{casos}^{(22,24)}$, havendo necessidade de uma ampla vitrectomia posterior na grande maioria deles para a reabilitação visual.

Devido ao fato destes olhos serem pseudofácicos ou afácicos e apresentarem múltiplas cirurgias prévias e processo inflamatório prévio e/ou crônico, não é rara a presença de edema macular cistóide, o que compromete também o prognóstico visual destes casos.

O glaucoma é uma complicação frequente e também ameaçadora do prognóstico visual em olhos tratados com KPro. Na maioria das vezes, a própria doença base é responsável pelo risco aumentado desta complicação, e o glaucoma refratário já existe previamente ao implante da ceratoprótese. O implante valvular de Ahmed tem sido a alternativa terapêutica de escolha para estes casos, com bom controle pressórico ${ }^{(4)}$.

A dificuldade pré-operatória de avaliação do nervo óptico, por opacidade de meios, e da pressão intraocular, pelas alterações da superfície corneana, subestimam as taxas de glaucoma pré-operatório, comprometendo o prognóstico desses pacientes e obrigando, muitas vezes, a necessidade de uma nova cirurgia para implante de drenagem.

Como não é possível uma tonometria nestes olhos após o implante da ceratoprótese, o acompanhamento do glaucoma se faz mediante a documentação fotográfica do nervo óptico e pela campimetria. A papilografia é mais difícil de ser realizada através dos $3 \mathrm{~mm}$ transparentes centrais da ceratoprótese, mas plenamente possível. Já a campimetria pode avaliar em média os $60^{\circ}$ centrais, o que costuma ser suficiente para a avaliação glaucomatosa.

Nossa pequena série de 28 olhos implantados com a Boston KPro tipo 1 revelou melhora da AV para uma visão funcional (20/200 ou melhor) em 64,3\% dos casos, durante um 
seguimento médio de 31,0 $\pm 21,2$ meses ( 2 a 72 meses). As complicações em nossa série de casos foram:

- necrose da interface em $21,4 \%$ dos casos, com necessida de da troca da córnea doadora (e reposicionamento da KPro) em todos menos um, onde o enxerto de membra na amniótica e o recobrimento foram suficientes para a cura do processo necrosante. Em todos os casos onde a córnea doadora foi trocada, e reposicionada a KPro, houve manutenção da acuidade visual, exceto em um paciente que foi a phthisisbulbi;

- vitreíte estéril em $25 \%$ dos casos, todos recuperados após vitrectomia posterior;

- opacidade de cápsula posterior em $25 \%$ dos casos, todos bem sucedidos após a realização de capsulotomia posterior com YAG laser;

- edema macular cistóide em 21,4\% dos casos, com tratamento clínico em $50 \%$ e com injeção intravítrea de triancinolona em $50 \%$;

- glaucoma em 17,8\% dos casos, todos controlados com implante de válvula de Ahmed e com tratamento clínico;

- membrana retroprotética em 10,7\% dos casos, tratados com injeção de ativador do plasminogênio tecidual (tPA) e/ou corticóide tópico e sistêmico;

- ceratite micótica em 14,3\%; um paciente foi tratado clinicamente e o outro com a retirada da córnea doadora e injeção intraocular de voriconazol.

A ceratoprótese de Dohlman é, portanto, uma importante alternativa para pacientes de alto risco para rejeição de transplante de córnea, com resultados bastante favoráveis a curto e médio prazo. Deve-se, no entanto às possíveis complicações a médio e longo prazo, que podem comprometer de maneira significativa o prognóstico visual destes pacientes.

\section{REFERÊNCIAS}

1. Strampelli B, Marchi V. [Osteo-odonto-keratoprosthesis]. Ann Ottalmol Clin Ocul. 1970;96(1):1-57.

2. Falcinelli G, Falsini B, Taloni M, Colliardo P, Falcinelli G. Modified osteo-odonto-keratoprosthesis for treatment of corneal blindness: long-term anatomical and functional outcomes in 181 cases. Arch Ophthalmol. 2005;123(10):1319-29.

3. Ngakeng V, Hauck MJ, Price MO, Price FWJr. AlphaCor keratoprosthesis: a novel approach to minimize the risks of longterm postoperative complications. Cornea. 2008;27(8):905-10.

4. Dohlman CH. Results of 218 eyes with keratoprosthesis. ASCRS Meeting. San Diego, USA, 2007

5. Ma JJ, Graney JM, Dohlman CH. Repeat penetrating keratoplasty versus the Boston keratoprosthesis in graft failure. Int Ophthalmol Clin. 2005;45(4):49-59.

6. Aquavella JV, Qian Y, McCormick GJ, Palakuru JR. Keratoprosthesis: the Dohlman-Doane device. Am J Ophthalmol. 2005;140(6):1032-8.

7. Aquavella JV, Qian Y, McCormick GJ, Palakuru JR Keratoprosthesis: current techniques. Cornea. 2006;25(6):656-62.

8. Dohlman, CH. Personal communication.American Society of Cataract and Refractive Surgery Meeting 2011. San Diego, CA. March 25-29, 2011

9. Stolz AP, Kwitko S, Dal Pizzol MM, Marinho D, Rymer S. Experiência inicial com a ceratoprótese de Dohlman-Doane: relato de casos. Arq Bras Oftalmol.2008;71(2):257-61.
10. Doane MG, Dohlman $\mathrm{CH}$, Bearse G. Fabrication of a keratoprosthesis. Cornea. 1996;15(2):179-84.

11. Klufas MA, Colby KA. The Boston keratoprosthesis. Int Ophthalmol Clin. 2010;50(3):161-75.

12. Barnes SD, Dohlman CH, Durand ML. Fungal colonization and infection in Boston keratoprosthesis. Cornea. 2007;26(1):9-15.

13. Moshirfar M, Marx DP, Kumar R. The effect of the fourth-generation fluoroquinolones on corneal reepithelialization after penetrating keratoplasty. Cornea. 2005;24(7):833-6.

14. Tsubota K, Goto E, Shimmura S, Shimazaki J. Treatment of persistent corneal epithelial defect by autologous serum application. Ophthalmology. 1999;106(10):1984-9.

15. Hicks CR, Crawford GJ. Melting after keratoprosthesis implantation: the effects of medroxyprogesterone. Cornea. 2003;22(6): 497-500.

16. Chiou AG, Florakis GJ, Kazim M. Management of conjunctival cicatrizing diseases and severe ocular surface dysfunction. Surv Ophthalmol. 1998;43(1):19-46.Review.

17. Tuft SJ, Shortt AJ. Surgical rehabilitation following severe ocular burns. Eye (Lond). 2009;23(10):1966-71.

18. Dogru M, Tsubota K. Current concepts in ocular surface reconstruction. Semin Ophthalmol. 2005;20(2):75-93.

19. Dua HS, Saini JS, Azuara-Blanco A, Gupta P. Limbal stem cell deficiency: concept, aetiology, clinical presentation, diagnosis and management. Indian J Ophthalmol. 2000;48(2):83-92.Comment in Indian J Ophthalmol. 2000;48(2):79-81.

20. Biber JM, Skeens HM, Neff KD, Holland EJ. The cincinnati procedure: technique and outcomes of combined living-related conjunctival limbal allografts and keratolimbal allografts in severe ocular surface failure. Cornea. 2011;30(7):765-71.

21. Meller D, Pires RT, Mack RJ, Figueiredo F, Heiligenhaus A, Park WC,et al. Amniotic membrane transplantation for acute chemical or thermal burns. Ophthalmology. 2000;107(5):980-9; discussion 990.

22. Aldave AJ, Kamal KM, Vo RC, Yu F. The Boston type I keratoprosthesis: improving outcomes and expanding indications. Ophthalmology. 2009;116(4):640-51.

23. Bradley JC, Hernandez EG, Schwab IR, Mannis MJ. Boston type 1 keratoprosthesis: the university of california davis experience. Cornea. 2009;28(3):321-7.

24. Zerbe BL, Belin MW, Ciolino JB; Boston Type1 Keratoprosthesis Study Group. Results from the multicenter Boston Type1 Keratoprosthesis Study. Ophthalmology. 2006;113 (10):1779.e1-7.

25. Chew HF, Ayres BD, Hammersmith KM,Rapuano CJ, Laibson PR, Myers JS, et al. Boston keratoprosthesis outcomes and complications. Cornea. 2009;28(9):989-96.

26. Yaghouti F, Nouri M, Abad JC, Power WJ, Doane MG, Dohlman $\mathrm{CH}$. Keratoprosthesis: preoperative prognostic categories. Cornea. 2001;20(1):19-23.

27. Dohlman, CH. Personal communication.American Society of Cataract and Refractive Surgery Meeting 2009. Las Vegas, USA, 2009.

28. Fintelmann RE, Maguire JI, Ho AC, Chew HF, Ayres BD. Characteristics of endophthalmitis in patients with the Boston keratoprosthesis. Cornea. 2009;28(8):877-8.

29. Durand ML, Dohlman CH. Successful prevention of bacterial endophthalmitis in eyes with the Boston keratoprosthesis. Cornea. 2009;28(8):896-901.

\section{Autor correspondente:}

Sérgio Kwitko

OftalmoCentro

Av. Nilo Peçanha, no 724/401

CEP 90.470-000 - Porto Alegre (RS), Brasil

E-mail: sergio@oftalmocentro.com.br 